\title{
Preface: Joint Discussion JD5 From Meteors and Meteorites to their Parent Bodies: Current Status and Future Developments
}

The Joint Discussion 5 entitled "From Meteors and Meteorites to their Parent Bodies: Current Status and Future Developments" within the IAU GA 2012 was organized with the coordination of the IAU Division III Planetary Systems Sciences and the IAU Commission N. 22 Meteors, Meteorites \& Interplanetary Dust, together with the supports by Divisions I Fundamental Astronomy, Division XII Union-Wide Activities, Commission 4 Ephemerides, Commission 6 Astronomical Telegrams, Commission 8 Astrometry, Commission 15 Physical Study of Comets \& Minor Planets, and Commission 20 Positions \& Motions of Minor Planets, Comets \& Satellites.

This Joint Discussion was aimed to share the latest knowledge on the small solar system bodies and also the possible parent bodies of meteors, meteorites \& interplanetary dusts from as wide a perspective as possible. The latest results were expected to come from international campaigns of ground-based observations (2011 Draconids meteor shower, fireball network etc.), space missions to comets \& asteroids (HAYABUSA, DAWN, EPOXI, Post-Stardust, Rosetta etc.) and meteorite falls \& recoveries. The scope extended to the meteor showers, meteorite falls, and comet appearance recorded in this oriental region over centuries. One of the purpose of this JD was to be dedicated to late Brian Marsden, who served as a leader of this field long time, and special invited talk on his work was set. We had 11 invited talks, 15 oral contributions along with 16 poster presentations in a wide range, from August 22 through 24. The most active researchers in this area attended the Joint Discussion and discussed the latest developments especially from Asian region. We wish to thank all participants to the meeting for their enthusiasm and for sharing their most recent results. I wish to thank the Scientific Organizing Committee for its advice and help in organizing the scientific program. The SOC ( ${ }^{*}$ Co-chairs) was composed of

Michael AfHearn (United States), Peter Brown (Canada), Tadeusz Jopek (Poland), Karen Meech (United States), Sho Sasaki (Japan), Caroline Smith (United Kingdom), Mitsuru Soma (Japan), Pavel Spurny (Czech Republic), Jeremie Vaubaillon (France), Hitoshi Yamaoka (Japan), Makoto Yoshikawa (Japan), Hajime Yano (Japan), Masateru Ishiguro (Republic of Korea), Daisuke Kinoshita (China Taipei), * Peter Jenniskens (United States), * Jun-ichi Watanabe (Japan), *Iwan Williams (United Kingdom), * Jin Zhu (China Nanjing).

I would like to thank Dr. Reiko Furusho for her kind effort to maintain the WEB site of our JD5 in the National Astronomical Observatory of Japan.

Junichi Watanabe

National Astronomical Observatory of Japan

email: jun.watanabe@nao.ac.jp 